\title{
Research on Multiple-Objectives Stochastic Dominance for Multiple Attribute Decision Making*
}

\author{
Lei WANG
}

Department of Economic and Management, Lanzhou JiaoTong University, Lanzhou, Gansu, China (wanglei@ mail.Lzjtu.cn)

\begin{abstract}
In view of multiple attribute decision making with random variables, in order to highlight research of multiple-objectives decision making, a method of decision making of stochastic dominance for the ranking of alternatives with multiple purpose based on Attribute Hierarchical Model is presented in this paper. In the basis of survey of present research of stochastic dominance method for stochastic multiple attribute decision making and analysis of stochastic dominance method and attribute hierarchical model, feasibility and concrete algorithm are pointed out for employing attribute hierarchical model in the stage of the ranking of alternatives based on dominance degree matrix of pairs of alternatives of every random attribute. The feasibility and validity of the proposed method are illustrated by a numerical example.
\end{abstract}

Keywords-multiple attribute decision making; stochastic dominance degree; attribute hierarchical model; multiple purpose decision making

\section{随机多属性决策的多目标随机占优方法研究}

\author{
王蕾 \\ 兰州交通大学经济管理学院, 兰州, 甘肃, 中国
}

摘 要 针对具有随机变量的多属性决策问题, 凸显多目标决策的研究目的, 提出了一种基于属性层次模型的随机占优决策方法 用于多目标方案优选排序。本文在对随机多属性决策环境下随机占优方法研究现状评述的基础上, 通过分析随机占优方法与属性层 次模型, 指出在根据每个随机属性的两两方案间的占优度矩阵得到方案的优选排序的阶段运用属性层次模型的可行性与具体算法, 算例结果表明本文所提方法的可行性与有效性。

关键词 多属性决策; 随机占优度；属性层次模型；多目标决策

\section{1. 现有主要研究分析}

多属性决策在经济、管理等领域中被广泛使用, 但目 前较为成熟的求解方法多要求事先确知方案的属性值。但 由于客观事物的复杂性和人类认识能力的局限性, 属性具 备不确定性的多属性决策问题的研究尚不多见; 随机性作 为不确定性的主要表现形式之一，随机多属性决策的研究 也尚具备较大研究空间, 在 CNKI 里以 “随机” 和 “多属性 决策” 为篇名关键字，在核心期刊仅检索到十余篇论文。

随机多属性决策是指在决策所依据的多个属性为随机 变量的场景下有限方案的选择问题。20 世纪 90 年代以 Zaras 等提出的 MSDr (Multi Attribute Stochastic
Dominance for A Reduced Number of Attributes) 概念 为基础, 随机占优方法被引入到多属性决策问题中。多数 探究集中在方案排序的相关研究中: Zaras 以粗糙集理论 为工具给出了随机多属性决策的方案排序模型; Nowak 提 出了基于随机占优准则和 ELECTRE III 方法 ${ }^{[1]}$ 相结合的 ${ }^{[2]}$ 和基于交互的方案排序方法 ${ }^{[3-4]}$; Yao 等在 Zaras 研究成果 的基础上, 将偏好概率进行分级, 并应用粗粘集方法进行方 案的选择 ${ }^{[5]}$ 。

上述研究在具体应用中经常存在以下两类问题: (1) 难于确定两两方案之间随机占优关系的强弱程度; (2) 无法 判定两两方案之间是否具有随机占优关系。这两类问题造

*国家自然科学基金项目支持（资助号：71461017），兰州交通大学青年科学基金支持（资助号：2011045） 
成的随机占优关系的信息缺失进而可能会影响最终的方案 优选，导致基于随机占优方法的多属性决策实用性降低。 国内外学者的相关改进研究主要集中:

(1) 引入随机占优准则来判断两两方案间在特定随机 属性是否存在占优关系, 但不同文献的随机占优准则存在 差异, 主要包括各阶随机占优准则和各阶近似随机优势准 则。

(2) 引入了占优度或相近概念来描述两两方案间在特 定随机属性上随机占优关系的强弱程度, 并进而构建针对 不同属性的两两方案间占优度程度矩阵。不同文献中占优 度概念定义及计算公式上虽然都表现为比例或对比关系, 但具体形式上存在差异。

随机多属性决策问题相关符号定义如下: $A\left(A, A, \ldots ., A_{m}\right)$ 为 $\mathrm{m}$ 个备选方案的集合; $C=\left(C_{1}, C_{2}, \ldots . ., C_{n}\right)$ 为 $\mathrm{n}$ 个随机属性且相互独立; 属性权 重向量为 $\left(w w_{2}, \ldots . ., w_{n}\right)$, 且满足 $\sum_{i=1}^{n} w_{i}=1$ 和 $w_{i} \geq 0, i=1,2, \ldots . n$; 决策矩阵 $X=\left\lfloor x_{i j}\right\rfloor_{m \times n}$ 中 $x_{i j}$ 表示方案 $\mathrm{A}_{\mathrm{i}}$ 对应于属性 $\mathrm{C}_{\mathrm{j}}$ 的随机评价结果，为区间 $\left[a_{j}, b_{j}\right]$ 上的随机 变量, 其累积分布函数为 $\mathrm{F}_{\mathrm{ij}}(\mathrm{x})$; 若 $F_{i j}(x) \neq F_{k j}(x)$,

$$
\begin{aligned}
\Omega_{i k}^{j}=\left\{x \mid F_{i j}(x)<F_{k j}(x), x \in\left[a_{j}, b_{j}\right]\right\}, \\
d_{i k}^{j}=\frac{\int_{\Omega_{k}^{j}}\left(F_{k j}(x)-F_{i j}(x)\right) d x}{\int_{a_{j}}^{b_{j}}\left|F_{k j}(x)-F_{i j}(x)\right| d x}
\end{aligned}
$$

(3) 由两两方案间在各个随机属性上的占有度矩阵如 何得到两两方案间的总体占优度矩阵, 目前基本采用的方 法都是简单加权法则，权值设定多为人工设定。

$$
d_{i k}=\sum_{j=1}^{n} w_{j} d_{i k}^{j}, i=1,2, \ldots, m, k=1,2, \ldots, m
$$

(4) 由两两方案间的占优度矩阵如何得到方案的优选 排序, 目前采用的主要方法如下:

(1)PROMETHEE II 方法, 其首先计算 Ai 的输出流 $\sum_{i=1}^{m} d_{i t}$ 和输入流 $\sum_{i=1}^{m} d_{t i}$, 然后以输出流和输入流的差值作为方案优 选排序的依据。

(2)与 PROMETHEE II 方法以绝对值为排序依据不同, 其以 $\sum_{i=1}^{m} d_{i t} \quad$ 这个相对值作为方案优选排序的依据。

$$
\overline{\sum_{i=1}^{m} d_{i t}+\sum_{i=1}^{m} d_{t i}}
$$

随机多属性决策中自然存在多个属性, 但上述研究中 仅将其作为属性来看待, 未能上升到决策目标的高度; 因 而在研究中依据每个属性的两两方案间的占优度矩阵得到
两两方案间总体占优度矩阵时, 采用的基本方法为简单加 权法则 (公式 2), 未能对此过程进行进一步的深入研究。 本文认为在每个随机属性上均存在不同的决策目标, 将决 策所依据的属性上升到决策目标的高度，借鉴属性层次模 型 (Attribute Hierarchical Mode1: AHM) 中的球赛模型 来建立方案的多目标决策模型。

\section{2. 属性层次模型 (AHM) 的简介}

为丰富与补充基于随机占优度方法的随机多属性决策 理论, 凸显多目标决策, 结合运筹学模型中的球赛模型, 本文进行了基于属性层次模型的随机占优度方法的多目标 决策研究。和以往研究不同, 根据两两方案间的总体占优 度矩阵得到方案的优选排序的方法借鉴的是运筹学模型中 的属性层次模型, 并同时在此过程中满足多目标决策的要 求。对属性层次模型的过程分析如下:

属性层次模型的基本模型为球赛模型: 设元素 $\mathrm{U}_{1}, \mathrm{U}_{2}$, $\mathrm{U}_{\mathrm{m}}$ 为 $\mathrm{m}$ 个球队, 球队 $\mathrm{U}_{\mathrm{i}}$ 和 $\mathrm{U}_{\mathrm{j}}(i \neq j)$ 之间进行比赛, 球队 $\mathrm{U}_{\mathrm{i}}$ 得分为 $u_{i j}$, 球队 $\mathrm{U}_{j}$ 得分为 $u_{j i}, u_{i i}=0$ (即球队不能和 自己比赛), 准则 $\mathrm{C}$ 为得分高低, 现要求按照准则 $\mathrm{C}$ 对 $\mathrm{n}$ 个 球队进行排序; 如 $u_{i j} \geq u_{j i}$ 则称 $\mathrm{U}_{\mathrm{i}}$ 比 $\mathrm{U}_{j}$ 强, 记为 $u_{i} \geq u_{j}$; 如满足 $u_{i j}+u_{j i}=1$ 则称 $\mathrm{u}_{\mathrm{i} j}$ 为相对属性测度; 由所有 $u_{i j}$ 构 成的矩阵 $\left(u_{i j}\right)$ 称为属性判断矩阵; 若属性判断矩阵 $\left(\mathrm{u}_{\mathrm{i} j}\right)$ 满足 $u_{i} \geq u_{j}, u_{j} \geq u_{k} \Rightarrow u_{i} \geq u_{k}$, 则称属性判断矩阵 $\left(\mathrm{u}_{\mathrm{ij}}\right)$ 具有一致性;

本文认为随机占优方法中相关概念与属性层次模型的 球赛模型中的要素具有相当的对应特性, 主要体现如下:

(1) 球队可以对应于待优选排序的方案;

(2) $u_{i j} \geq u_{j i}$ 则称 $\mathrm{U}_{\mathrm{i}}$ 比 $\mathrm{U}_{j}$ 强对应于方案 $\mathrm{i}$ 随机占优于 方案 $j$;

(3) $u_{i i}=0$ (即球队不能和自己比赛) 对应于方案 $\mathrm{A}_{\mathrm{i}}$ 与 其自身之间不存在随机占优关系;

(4) 准则 $C_{1} 、 C_{2} 、 \cdots 、 C_{n}$ 对应于多目标的 $n$ 个目标准则;

(5) 由公式 (1) 计算得到的两两方案间总体占优度也符 合相对属性测度和属性判断矩阵的定义;

（6）由上述定义易证，由公式 1 计算得到的两两方案间 总体占优度矩阵具备一致性特性。 
由于随机占优方法和球赛模型存在的特定对应关系, 因而完全可以使用属性层次模型的方法来根据两两方案间 总体占优度矩阵来得到多目标情景下的方案优选排序。

\section{3. 基于属性层次模型 (AHM) 的随机占优度方法与具体 算例}

由于公式 (1) 所得两两方案间总体占优度更为符合属 性层次模型 (AHM), 因此下述随机占优方法以公式 (1) 为依 据来计算两两方案间总体占优度, 并判断两两方案间占优 关系。具体步骤如下:

(1) 依据公式 (1) 计算并建立针对每个属性的两两方案 间的占优度矩阵;

(2) 依据公式 (3) 计算属性层次模型中各准则的相对属 性权;

准则 $C_{\mathrm{i}}$ 相对属性权: $W_{C_{i}}=\left(\begin{array}{llll}w_{C_{i} 1} & w_{C_{i} 2} & \ldots & w_{C_{i} m}\end{array}\right)$,

$$
w_{c_{i} j}=\frac{2}{m(m-1)} \sum_{j=1}^{m} u_{i j}
$$

其中 $\mathrm{m}$ 为备选方案的数量, $u_{i j}$ 为方案 $\mathrm{i}$ 相对于方案 $\mathrm{j}$ 的随机占优度, 准则 $\mathrm{C}_{\mathrm{i}}$ 相对属性权反应了在准则 $\mathrm{C}_{\mathrm{i}}$ 下各个 备选方案的总体占优程度, 可证准则 $\mathrm{C}_{\mathrm{i}}$ 下各个备选方案的 总体占优程度之和为 1 ;

(3) 验证每个属性的两两方案之间的占优度矩阵是否 符合相对属性测度的定义，验证其是否具备一致性特性;

(4) 根据给定的属性权重, 依据公式 4 计算得到属性判 断矩阵 $\left(\mathrm{G}_{\mathrm{i} j}\right)$, 由公式 4 可知属性判断矩阵符合相对属性测 度的定义和具备一致性特性;

$$
G_{i j}=\left\{\begin{array}{rr}
\frac{w_{i}}{w_{i}+w_{j}} & w_{i}>w_{j} \\
0.5 & w_{i}=w_{j} \\
\frac{w_{j}}{w_{i}+w_{j}} & w_{i}<w_{j}
\end{array}\right\}
$$

其中 $W$ 为决策者提供的属性权重向量;

与公式 3 类似, 属性判断矩阵的相对属性权 $\mathrm{W}_{\mathrm{G}}$ 为:

$$
W_{G}=\left(\begin{array}{llll}
w_{G 1} & w_{G 2} & \ldots & w_{G n}
\end{array}\right), \quad w_{G i}=\frac{2}{n(n-1)} \sum_{j=1}^{n} G_{i j}
$$

其中 $\mathrm{n}$ 为评价指标个数;

(5) 依据公式 5 计算方案对总目标的合成权重, 以此为 基础形成方案的优选序列。

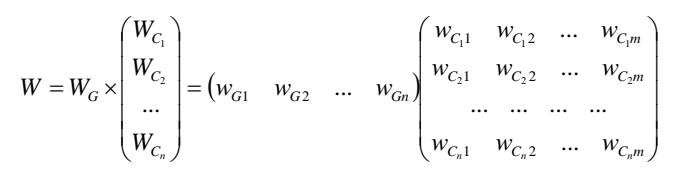

具体算例为多属性供应商选择问题: 有 5 个备选供应
商 $\left(A_{1}, A_{2}, A_{3}, A_{4}, A_{5}\right), 3$ 个评价指标 $\left(C_{1}, C_{2}, C_{3}\right)$ 分别表 示货物质量水平、售后服务水平和货物价格, 存在三个与 此对应的决策目标; 决策者聘请 7 位专家采用 10 分制形式 (1 分表示最差, 10 分表示最好) 来评价每个供应商针对各

\begin{tabular}{|c|c|c|c|c|c|c|}
\hline \multirow{2}{*}{ 指标 } & \multirow{2}{*}{ 分值 } & \multicolumn{5}{|c|}{ 方案 } \\
\hline & & $\mathrm{A}_{1}$ & $\mathrm{~A}_{2}$ & $\mathrm{~A}_{3}$ & $\mathrm{~A}_{4}$ & $\mathrm{~A}_{5}$ \\
\hline \multirow{8}{*}{$\mathrm{C}_{1}$} & 1 & & & $1 / 7$ & & \\
\hline & 2 & & $1 / 7$ & & & $1 / 7$ \\
\hline & 3 & $1 / 7$ & & & $1 / 7$ & \\
\hline & 4 & $3 / 7$ & $2 / 7$ & & $1 / 7$ & \\
\hline & 5 & $2 / 7$ & $1 / 7$ & $3 / 7$ & $1 / 7$ & \\
\hline & 6 & & $2 / 7$ & & $3 / 7$ & $2 / 7$ \\
\hline & 7 & & & $1 / 7$ & $1 / 7$ & $2 / 7$ \\
\hline & 8 & $1 / 7$ & $1 / 7$ & $2 / 7$ & & $2 / 7$ \\
\hline \multirow{10}{*}{$\mathrm{C}_{2}$} & 1 & & & & $1 / 7$ & \\
\hline & 2 & & $1 / 7$ & & & $1 / 7$ \\
\hline & 3 & $2 / 7$ & & & & $1 / 7$ \\
\hline & 4 & & & $3 / 7$ & $1 / 7$ & \\
\hline & 5 & $2 / 7$ & & $1 / 7$ & & \\
\hline & 6 & & $1 / 7$ & & $1 / 7$ & $2 / 7$ \\
\hline & 7 & $1 / 7$ & $1 / 7$ & & & \\
\hline & 8 & $1 / 7$ & $4 / 7$ & $2 / 7$ & $3 / 7$ & $2 / 7$ \\
\hline & 9 & $1 / 7$ & & & $1 / 7$ & $1 / 7$ \\
\hline & 10 & & & $1 / 7$ & & \\
\hline \multirow{7}{*}{$\mathrm{C}_{3}$} & 1 & & & & & $1 / 7$ \\
\hline & 2 & $1 / 7$ & & & & \\
\hline & 3 & & $2 / 7$ & & $1 / 7$ & \\
\hline & 4 & & & $3 / 7$ & $2 / 7$ & \\
\hline & 5 & $2 / 7$ & $4 / 7$ & $3 / 7$ & $4 / 7$ & $2 / 7$ \\
\hline & 6 & & $1 / 7$ & $1 / 7$ & & $3 / 7$ \\
\hline & 7 & $4 / 7$ & & & & $1 / 7$ \\
\hline
\end{tabular}
指标情况, 概率分布形式的评价结果如表 1 所示。下面说 明基于属性层次模型 (AHM) 的随机占优度方法计算过程。

表 1 评价结果的概率分布表

(1) 依据公式 1 计算并建立针对每个属性的两两方案 间比较的占优度矩阵，其中 “-” 表示不存在占优关系

目标准则 $\mathrm{C}_{1}$ 两两方案间占优度矩阵:

$$
D_{1}=\left|\begin{array}{ccccc}
- & 1 / 4 & 1 / 5 & 1 / 6 & 1 / 13 \\
3 / 4 & - & 1 / 6 & 1 / 4 & 0 \\
4 / 5 & 5 / 6 & - & 5 / 8 & 0 \\
5 / 6 & 3 / 4 & 3 / 8 & - & 1 / 9 \\
12 / 13 & 1 & 1 & 8 / 9 & -
\end{array}\right|
$$

目标准则 $\mathrm{C}_{2}$ 两两方案间占优度矩阵:

$$
D_{2}=\left|\begin{array}{ccccc}
- & 2 / 11 & 1 / 5 & 1 / 4 & 1 / 4 \\
9 / 11 & - & 2 / 3 & 4 / 5 & 6 / 7 \\
4 / 5 & 1 / 3 & - & 4 / 9 & 4 / 7 \\
3 / 4 & 1 / 5 & 5 / 9 & - & 3 / 4 \\
3 / 4 & 1 / 7 & 3 / 7 & 1 / 4 & -
\end{array}\right|
$$

目标准则 $\mathrm{C}_{3}$ 两两方案间占优度矩阵: 


$D_{3}=\left|\begin{array}{ccccc}- & 9 / 10 & 9 / 11 & 10 / 11 & 1 \\ 1 / 10 & - & 1 / 3 & 2 / 3 & 1 / 4 \\ 2 / 11 & 2 / 3 & - & 1 & 1 / 3 \\ 1 / 11 & 1 / 3 & 0 & - & 2 / 9 \\ 0 & 3 / 4 & 2 / 3 & 7 / 9 & -\end{array}\right|$

（2）依据公式 3 计算属性层次模型的各目标准则的相 对属性权;

准则 $\mathrm{C}_{1}$ 下相对属性权:

$w_{C_{1}}=\left(w_{C_{1} 1}, w_{C_{1} 2}, \ldots, w_{C_{1} m}\right)=(0.0694,0.1167,0.2258,0.2$

069, 0. 3812)

准则 $\mathrm{C}_{2}$ 下相对属性权:

$w_{C_{2}}=\left(w_{C_{2} 1}, w_{C_{2} 2}, \ldots, w_{C_{2} m}\right)=(0.0882,0.3142,0.2149,0$

$.2256,0.1571)$

准则 $\mathrm{C}_{3}$ 下相对属性权:

$w_{C_{3}}=\left(w_{C_{3} 1}, w_{C_{3} 2}, \ldots, w_{C_{3} m}\right)=(0.3627,0.1350,0.2182,0$.

0646, 0. 2194)

(3) 验证每个随机属性的两两方案间的占优度矩阵是 否符合相对属性测度和定义, 验证其是否具备一致性特性;

易看出每个随机属性的两两方案间的占优度矩阵符合 相对属性测度的定义，并且具备一致性特性。

(4) 计算属性判断矩阵及其相对属性权, 下面以两个不 同属性权重向量分别计算属性判断矩阵及其相对属性权:

(1)假设决策者提供的属性权重向量 $\mathrm{w}=(1 / 3,1 / 3$, $1 / 3)^{\mathrm{T}}$, 以此权重向量计算主要用于和以往算例进行对比。

依据公式 4 计算属性判断矩阵为:

$$
G=\left(\begin{array}{ccc}
0 & 0.5 & 0.5 \\
0.5 & 0 & 0.5 \\
0.5 & 0.5 & 0
\end{array}\right)
$$

依据公式 5 计算属性判断矩阵的相对属性权为: $W_{G}=(1 / 3,1 / 3,1 / 3)$; 由此可见当指标权重相等时，属性 判断矩阵的相对属性权等同于指标权重。

(2)假设决策者提供的属性权重向量 $\mathrm{w}=(0.6,0.3$, $0.1)^{\mathrm{T}}$, 此反映在选择供应商时偏重于产品质量与售后服务 质量, 相对对于价格不够敏感。

依据公式 4 计算属性判断矩阵

为: $G=\left(\begin{array}{ccc}0 & 0.6667 & 0.8571 \\ 0.3333 & 0 & 0.75 \\ 0.1429 & 0.25 & 0\end{array}\right)$, 依据公式 5 计算属性判断矩阵 的相对属性权为: $W_{G}=(0.5079,0.3611,0.1310)$ 。
（5）计算方案对总目标的合成权重, 以此为基础形成方 案的优选序列。

下面对算例步骤 (4) 中两个属性权重向量分别计算方 案对目标的合成权重:

(1)依据公式 6 计算方案对目标的合成权重为: $\mathrm{W}=(0.1734,0.1886,0.2196,0.1656,0.2525)$

由此可得方案优选排序结果为:

$A_{5} \geq A_{3} \geq A_{2} \geq A_{1} \geq A_{4}$; 此方案优选排序结果与采用

PROMETHEE II 方法所得方案优选排序结果相同。

(2) $W=(0.1146,0.1904,0.2209,0.1950,0.2791)$

由此可得方案优选排序结果为:

$A_{5} \geq A_{3} \geq A_{4} \geq A_{2} \geq A_{1}$; 与相同权重的方案优选排序结果相

比, 方案 4 排序顺序存在较大变化, 源于方案 4 在属性权 重高的 $C_{1} 、 C_{2}$ 属性中高分概率高于方案 2 和方案 1 , 而在 在属性权重低的 $\mathrm{C}_{3}$ 属性中高分概率低于方案 2 和方案 1 , 排序结果合理。

\section{4. 结语}

上述理论分析与算例结果表明本文提出的基于属性层 次模型的多目标随机占优度方法的可行性, 丰富与补充了 依据每个随机属性的两两方案间的占优度矩阵得到方案的 优选排序的阶段中可以采用的算法。

\section{参考文献(References)}

[1] Roy B. Multicriteria methodology for decision aiding. Dordrecht: Kluwer Academic publishers, 1996.

[2] Nowak M. Preference and veto thresholds in multi-criteria analysis based on stochastic dominance. European Journal of Operational Research, 2004, 158: 339-350.

[3] Nowak M. INSDECM-an interactive procedure for stochastic multi-criteria decision problems. European Journal of Operational Research, 2006, 175: 1413-1430.

[4] Nowak M. Aspiration level approach in stochastic MCDM problems. European Journal of Operational Research, 2007, 177: 1626-1640.

[5] Yao S B, Yue C Y. Approach to stochastic multi-attribute decision problems using rough sets theory. Journal of Systems Engineering and Electronics, 2006, 17(1): 103-108. 